no clear evidence that spot positivity was different in the spring/ summer months compared with autumn/winter.

Conclusion Seasonal variation did not appear to account for the fluctuations in spot positivity observed. We conducted a sub-group analysis restricted to 60 year-olds to explore the possibility that changes in the demographics of participants over time might affect the positivity patterns, but the data yielded similar inconsistencies. It is unlikely that operational factors account for the fluctuations as the BCSP ensures rigorous monitoring of test kit batches and quality control in the laboratories. More work is required to explore these data further in search of an explanation.

Disclosure of Interest None Declared.

\section{PWE-021 AN INVESTIGATION INTO HIGH GFOBT POSITIVITY DURING THE FIRST FIVE DAYS FOLLOWING SAMPLE COLLECTION}

doi:10.1136/gutjnl-2013-304907.310

1,2, H E Seaman, 1J M Snowball, 1,2S P Halloran. 'NHS Bowel Cancer Screening Programme, Southern Hub; ${ }^{2}$ University of Surrey, Guildford, UK

Introduction The NHS Bowel Cancer Screening Programme (BCSP) in England has used the guaiac-based faecal occult blood test (gFOBt) since 2006. Test positivity is higher during the first 5 days after sample collection.

Methods The gFOBt kits used by the BCSP have six windows (three pairs) lined with guaiac-impregnated philtre paper. The screening participant is asked to apply two faecal samples from three separate stools. The samples usually arrive dry at the laboratory, which is likely to reduce the degree of haemoglobin $(\mathrm{Hb})$ degradation and provide the best opportunity to detect bleeding. BCSP policy is that every kit is logged on the day of receipt and read as soon as possible thereafter. BCSP Southern Hub data from the Bowel Cancer Screening System (BCSS) for 01/2008-07/2012 were analysed for subjects aged 60-69 years completing the first prevalent round of screening. The interval between each sample date and the date the sample was analysed (elapsed time) was calculated. Spot positivity was assessed by elapsed time, stratified by sex. Clinical outcomes were extracted from BCSS for subjects who had a positive gFOBt result. The relationship between positivity, elapsed time and clinical outcomes was examined.

Results During the period of observation, nearly 1.2 million kits were returned to the Hub and $92 \%$ were read within five days of the last sample collection. For samples analysed one day after the last sample collection, spot positivity was $2.7 \%$ for women and $3.8 \%$ for men. Positivity declined thereafter until it reached a steady level at day 5 (women $1.3 \%$; men $2.0 \%$ ). Positivity for the most recently collected sample (usually spots 5 and 6 ) was slightly higher than for the spots collected earlier. There were 20,408 subjects $(8,343$ women; 12,065 men) who accepted further investigation. The outcome of those investigations was not associated with elapsed time between faecal sampling and test kit reading; the proportion of cancers, high-, intermediate- and low-risk adenomas was fairly constant as elapsed time increased.

Conclusion The higher positivity associated with a shorter elapsed time did not appear to be associated with more false-positive tests. A number of factors may contribute to the pattern of positivity observed: (a) vegetable peroxidases present in the diet cause short term increases in gFOBt positivity; (b) faecal Hb denatures gradually before analysis, and (c) screening invitees worried about their health may return test kits more rapidly than others and a proportion of those individuals will be found to have colorectal cancer and related disease. None of those factors alone can account for the marked elevation in the 'early positivity rate' and further studies are required to elucidate the reasons for this effect. Disclosure of Interest None Declared.

\section{PWE-022 CHANGING LIFESTYLE TO REDUCE COLORECTAL CANCER IN A HIGH RISK POPULATION}

doi:10.1136/gutjnl-2013-304907.311

1.2," I A Murray, ${ }^{3} \mathrm{G}$ P Tarr, ${ }^{3} \mathrm{R}$ John, ${ }^{3} \mathrm{~A}$ Crowley, ${ }^{3} \mathrm{~J}$ Kok, ${ }^{3} \mathrm{H}$ Mustafa, ${ }^{3} \mathrm{H}-\mathrm{N}$ Lee, ${ }^{3} \mathrm{~L}$ Weaver, ${ }^{3} \mathrm{R}$ Smith, ${ }^{3} \mathrm{O}$ Son, ${ }^{3} \mathrm{~K}$ M Sii, ${ }^{3} \mathrm{C}$ Cameron, ${ }^{3} \mathrm{~J}$ Dockerty, ${ }^{3} \mathrm{M}$ Schultz. ${ }^{1}$ Gastroenterology, Royal Cornwall Hospital, Truro, UK; ${ }^{2}$ Gastroenterology, Dunedin Hospital; ${ }^{3}$ Dunedin School of Medicine, University of Otago, Dunedin, New Zealand

Introduction Both genes and environment are important contributors to colorectal cancer (CRC) risk. Modifying lifestyle has been shown to reduce CRC even in patients with higher genetic risk. Patient anxiety, risk perception and knowledge may affect healthy behaviour change. We examined these and their relation to selfreported lifestyle changes.

Methods 250 patients who had undergone surveillance colonoscopyfor a family history of CRC were randomly selected. A telephone interview was conducted. Demographics, family history, risk factor knowledge, perceptions of lifetime CRC risk, worry due to family history, and self-reported behavioural change due to thoughts about CRC were obtained. General anxiety was assessed using the GAD-7 scale. Ordinal logistics regression was performed to determine significance. Ethics approval was obtained.

Results 148 participants responded (69\%). Average age was 55.3 years, $96 \%$ were NZ European and over $80 \%$ had a lifetime risk of CRC of at least $18 \%$ (NZ Guidelines groups $2 \& 3$ ). Change in at least one lifestyle measure due to concerns about CRC was reported by $88.5 \%$. Dietary variables and physical activity were more likely to be changed (increased fibre, $63 \%$; increased fruit and vegetables, $54 \%$; reduced red meat, 47\%; more physical activity, 45\%), with consumption of tobacco, alcohol, and body weight less likely to be altered (25\%,26\% and 31\% respectively).

Adjusted odds ratios for the association of knowledge, anxiety and worry with adoption of healthy behaviours

\section{Abstract PWE-022 Table 1}

\begin{tabular}{llll}
\hline & $\begin{array}{l}\text { Adjusted } \\
\text { Odds Ratio* }\end{array}$ & $\begin{array}{l}\text { 95\% } \\
\text { Confidence } \\
\text { Interval }\end{array}$ & P value \\
\hline Perceived knowledge of CRCrisk factors & - & - & - \\
None & 3.76 & 0.76 to 18.7 & 0.11 \\
A little & 5.39 & 1.18 to 24.56 & 0.03 \\
Somewhat & 9.49 & 2.08 to 43.32 & 0.004 \\
Very much so & & & \\
Are genes the main cause of CRC? & - & - & - \\
Not at all & 1.79 & 0.36 to 9.02 & 0.48 \\
A little & 1.83 & 0.39 to 8.61 & 0.45 \\
Somewhat & 2.34 & 0.49 to 11.25 & 0.29 \\
Very much so & & & \\
Perceived risk of CRC, per SD increase. & 1.21 & 0.90 to 1.61 & 0.20 \\
Global GAD-7 score, per SD increase. & 1.51 & 1.12 to 2.03 & 0.006 \\
Worry about CRCdue to family history & - & - & - \\
Never & 2.93 & 1.05 to 8.13 & 0.038 \\
A little & 2.94 & 1.15 to 7.51 & 0.024 \\
Sometimes & 5.25 & 1.88 to 14.70 & 0.002 \\
Often/Alwayst & & & \\
\hline
\end{tabular}

* Adjusted for age, gender, and NZ Deprivation score.

Conclusion Most patients adopted at least one lifestyle change to reduce their risk. Those with higher anxiety levels, specific worry due to family history, and those who believed they knew more about CRC risk factors were more likely to report healthy lifestyle changes. Overall perceived risk of developing CRC and actual risk due to family history were not associated with behaviour change.

Disclosure of Interest None Declared. 